\title{
CONSUMER HEALTH PLAN DECISIONS
}

\author{
TRACY BLACK \\ JODI JENKINS
}

\author{
Graduate School of Public Health, A640 Crabtree Hall \\ University of Pittsburgh, Pittsburgh, PA 15261, U:S.A.
}

\begin{abstract}
We demonstrate the application of supermatrix feedback in consumer health plan decisions using value judgements based on importance of choice, quality, and costs, among other factors. This model not only enables consumers to prioritize benefit options, but also allows health plans to structure programs to meet consumer needs and desires.
\end{abstract}

\section{INTRODUCTION}

Important considerations in health plan decisions include: 1.) What benefits do consumers want to have covered in their health plan?; 2.) What information should be made available to aid consumers in health plan decisions?; and 3.) How can health care reform appease consumers' desires? The answers to such questions are difficult to determine as there is no consistent definition of quality health care. What is important to some individuals may not be important to others. ${ }^{1}$ In general, consumers equate quality with not only easily measurable factors but also personal experiences. These subjective factors include previous expectations, attitudes and beliefs, and degree of personal involvement in plan design. ${ }^{2}$ A major component in a person's sense of quality depends upon his or her ability to choose a provider and treatment at any given time. The outcomes of patient encounters with health care services, both in terms of satisfaction and quality perception, are influenced by the amount of consumer choice and loyalty to physician. ${ }^{3}$

In response to the integration of consumer needs for health care reform, the super-matrix model presented in this paper could be applied in the following ways:

- to aid consumers in their health plan decisions;

- to determine the degree of influence various factors have on consumer health plan decisions;

- to rank health plans according to their ability to meet consumer needs; and

- to assist health plan providers in the design of benefit coverage, accordingly.

Of course, this is only a partial list. Furthermore, a regional concept is particularly important given the local nature of consumer preferences and of health care delivery systems.

There also is a growing concern that many individuals are not covered by health insurance and may be foregoing necessary care. The consequences of delay are that problems worsen and become more expensive to treat. While several factors contribute to a consumer's decision not to purchase health benefits, prohibitive cost is not necessarily the primary reason.

Almost half of full-time workers in the U.S. are covered under traditional fee-for-service indemnity plans, however, a growing number of businesses are finding a promising avenue in managed care. Although still dominant among small and medium size employers, fee-for-service plans continue to lose market share. Managed care enrollment grew from $29 \%$ to $51 \%$ of employees among all sizes of firms between 1988 and 1993. ${ }^{4}$ While a company can only do so much to educate employees about the high cost of health care, more and more companies are 
selecting managed care plans because those carriers are knowledgeable about managing costs and encouraging hospitals and physicians to be the same. Although some consumers believe that managed care programs do not provide individuals opportunities to receive quality care, others have experienced differentiy. Before presenting the super-matrix model using the health benefit options offered by the University of Pittsburgh to its employees, a brief description and comparison of the various providers of health care benefits is necessary.

Managed care plans offered through health maintenance organizations (HMOs) and preferred provider organizations (PPOs) enable employers to maximize their dollars spent; offering health insurance to employees thus becomes more affordable. Additionally, managed care often provides greater coverage and more benefits (particularly wellness programs) for those enrolled in such plans. For example, virtually all HMOs provide well-baby coverage while only $50 \%$ of individuals in conventional plans receive such coverage. ${ }^{5}$ Similar disparities exist for other preventive services. Yet employers who do not include non-traditional health insurance options in their benefits packages are not just offering PPO riders (add-on benefits) to their indemnity plans. Firms are selecting HMOs, point-of-service (POS) plans, and combinations of these plans, as well. By offering a number of health benefit options, employers allow the employees to decide which health plan best fits their individual needs. Below is a description for each of the major types of provider plans:

- Indemnity insurance is traditional/conventional medical insurance where an insurance carrier reimburses individuals or their providers after the health care services have been rendered. Payments are made regardless of the source or location of the medical services, and there is no limit to the type and number of services the insured can receive.

- PPOs contract with providers for health care services at a discounted rate. The network is limited in size and usually has some form of utilization review system. The PPO may or may not be risk bearing and may or may not use a gatekeeper Primary Care Physician (PCP) who provides or arranges for all of the member's needs.

- HMOs provide an orgànized system which combines the delivery and financing of comprehensive health care services to voluntary enrolled subscribers for a fixed prepaid fee. HMOS manage health care costs by emphasizing preventive medicine, negotiating provider discounts, and using a utilization management program. One key element of HMO base health benefits includes the coverage of preventive and sickness at 100\%, whereas PPO base health benefits cover sickness only. HMOs also offer more riders, an important factor for those individuals with special health care needs.

- POS plans are similar to HMOs in that both require the insured to utilize a capitated PCP as a gatekeeper to coordinate medical care. However, the major difference between the POS and HMO plans is that the POS allows the insured to bypass the gatekeeper, self-refer to a specialist, and still receive some level of coverage for most services. As a result, POS plans are often referred to as an HMO plan that provides an out-of-plan or swing-out benefit. Although consumers do not have to choose a provider who will provide medical services until they need the services, a POS plan incorporates incentives for individuals to use participating (par) providers.

\section{DISCUSSION ON SUPER-MATRIX MODEL AND RESULTS}

The super-matrix consists of five primary factors/components (clusters) that have been determined to influence a consumer's health plan decision: provider networks, quality assurance, costs/expenses, rider benefits, and health plans. The importance of these clusters is 
prioritized in the cluster matrix. Provider networks is the most important cluster in the decision process because consumers are primarily concerned with their choice of available physicians and hospitals in any given plan. Choice is considered to be the most valued characteristic of traditional indemnity plans and contributes to the stigma generally associated with the most restrictive forms of managed care (staff HMOs). Quality assurance is the second most important cluster since consumers select health plans not only on provider networks but also on covered benefits. Consumers typically perceive quality in the preventive services, reputation, and past performance of each plan. Costs/expenses is the third most important cluster. Although consumers usually will pay anything when it comes to their health or the health of a loved one, costs/expenses are higher when the plan does not manage consumer utilization of providers and services. As a result, HMOs and P.POs are able to offer more benefits at lower costs to consumers compared to traditional indemnity plans due to utilization management techniques inherent to managed care plans. Rider benefits is the fourth most important cluster. While consumers would like to have every health service covered as a benefit, they realize that doing so is unreasonable and accept add-ons to plan options to cover their special health care needs. Finally, the least important cluster that impacts consumer health plan decisions is comprised of the choices and preference of health plans, themselves. After weighing values in order of importance, consumers will choose whatever plan best meets their particular health care needs to arrive at the final outcome.

For each cluster, there are a number of criteria/elements that more specifically influence the outcome/choice in consumer health plan decisions. The importance (value) of each element is determined by assessing our personal experiences as health care consumers, drawing on information from numerous articles and seminars quoted throughout this paper, and speaking with health care administration professionals, as indicated.

The provider network cluster has six elements, with the element most important to consumers being choice. Choice involves the number of physicians/health care providers from which consumers are able to choose, and the greater number of providers results in the greater ease of seeing a provider of choice. The freedom to be able to remain with one's present physician is also a characteristic of choice. This is crucial to consumers who do not want their choice of physicians limited. Limited choice is the major reason that many Americans do not readily accept the concept of managed care and choose to remain in a traditional indemnity insurance arrangement. The second most important element is the availability of medical care, which relates to the arrangements for choosing a personal provider. The third most important element involves whether or not expansion plans are included in the coverage. Again, consumers want the ability to be able to broaden their realm of service and provider choices. Expansion plans indicate the potential for the number of providers, thus choice, to increase. The fourth element is credentialing of the health care providers included in the plan. Credentialing involves 'the evaluation of providers' skills, historical performance, and training to ensure consumers receive thorough and accurate care. The fifth element is accessibility (arranging for and getting care). This element deals primarily with the convenience of provider locations or, in other words, the distance a consumer will have to travel to obtain care. The last element is affability, which involves the communication and interpersonal care demonstrated by providers. Although this element is ranked least important in the provider networks cluster, consumers still value friendliness and courtesy, respect and attention, reassurance and support, clear explanations of medical procedures and tests, and real time spend with health care providers.

The quality assurance cluster consists of five elements. The most important element in 
consumer health plan decisions is whether or not a plan provides coverage for preventive services. Consumers not only want plans that cover iliness visits, treatment, and hospitalization, but also preventive care and routine office visits. Consumers want to avoid illness and stay healthy. Such comprehensive coverage is particularly important to families with children. The second most important element is the reputation of the health plan, which tends to reflect outcomes/performance, the third most important element. The close inter-relatedness of these two elements is evident in the weights consumers placed on each, which were almost equal. Consumers increasingly are interested in the outcomes of medical care and the performance of providers as they evaluate benefits received. Member services is the fourth element and involves such benefits as access to educational materials to help manage or prevent medical problems. The element least important to consumers is claims processing. Although claims processing is an important consideration, consumers tend to weigh quality and access more heavily than the speed and convenience of the claims processing when making health plan decisions. However, consumers do place importance on the ease of completing few or no forms and the length of time spent on paperwork, in general. Because consumer experience with claims processing usually occurs through the member services area, the area consumers relay any questions or concerns about the way a plan processes forms and other paperwork needs to have knowledgeable and courteous staff to maintain favorable consumer relations.

There are five basic elements associated with costs and expenses of a health care plan that consumers evaluate in their health plan decisions. The amount of coinsurance split between the employer and the employee is the most important element because it has a direct impact on employee choice. If the employer contributes a much greater percentage of reimbursement for care rendered by par-providers versus non-par providers, the employee will be responsible for a larger financial portion of the bill if care was received by a non-par provider. Thus, the employer is able to steer the employee to par-providers with financial incentives to stay within the provider network rather than seeking care elsewhere. The second most important element involves the amount of co-payment required for health services. The inclusion of co-payments is very attractive to consumers because it reduces out-of-pocket expenses and claims paperwork. Co-payments for HMOs tend to range from only $\$ 2$ to $\$ 10$ for each office visit. The third most important element involves the bi-monthly contribution. This is the amount of premium paid by the employee for the covered benefits of the health plan regardless of whether or not care is received. The fourth element is deductible requirements. As total premium costs for all health plans have risen between 1988 and 1993, so have the average deductibles for traditional and PPO plans. Typically, trends in total premium costs and trends in cost-sharing are similar -they rise in tandem, although not necessarily at the same rates. HMOs typically do not require deductibles. The element least important to consumers is the out-of-pocket maximum that the consumer is subject to under the health plan. Out-of-pocket expenses not only include the amount paid for services excluded from coverage by a plan, but also any co-payments and deductibles required. Thus, out-of-pocket maximums reflect the dollar amounts required by each plan for other elements in the cost/expense cluster.

The rider benefits cluster has nine elements. The elements include a range of services which may be offered under a health care plan as add-on benefits. By far the most important elements are coverage for emergency room visits and prescription drugs, respectively. This reflects the potential for the consumer to incur extremely high costs generally associated with each of the two elements. Because of the great cost involved with emergency room visits, consumers are particularly aware of the need for this type of catastrophic coverage. Prescription drug coverage 
is also very important to most consumers because of rising pharmaceutical costs despite the influx of generic drugs. The third benefit most important to consumers is dental coverage. The fourth most important benefit is rehabilitative services, including physical therapy and substance abuse programs. Coverage for eye care is fifth most important to consumers. Mental health care coverage is ranked next in importance to consumers. The seventh rider benefit is coverage for medical equipment and devices, including crutches and wheelchairs. The last two elements are skilled nursing facilities and home health care. The weight of the last two elements really depends on specific health care needs of the particular consumer. Obviously, younger consumers will not attach the same values to these benefits as would be expected from elderly consumers. Actually, this concept applies throughout this cluster, as consumers weigh rider benefits options according to individual health care needs.

The last cluster involves the five health plans from which University of Pittsburgh employees can choose in order of preference - Traditional Blue Cross/Biue Shield/Major Medical Plan (BC/BS Traditional), HealthAmerica, Deductible Blue Cross/Blue Shield/Major Medical Plan, University Health Network (UHN) of University Health Services, Inc. (UHS), and Keystone West. Consumers' preference for traditional indemnity insurance plans and emphasis on the importance of choice is demionstrated by the fact that the traditional plan is the most preferred. There are no service area restrictions associated with the Traditional and Deductible Blue Cross/Blue Shield/Major Medical plans. For the other plans, approved provider and service area restrictions apply, but there are provisions for medically necessary specialists or out-of-area emergency care. Each of the five plans has different provisions for inpatient and outpatient mental health services, prescription drug services and other specialized services. Indicative of changing market forces, however, the Traditional Plản will no longer be available to University of Pittsburgh employees after 1994.

\section{CONCLUSIONS}

The $\mathrm{BC} / \mathrm{BS}$ Traditional Indemnity plan is the most likely plan to be chosen by consumers based on values placed on each element and cluster in relation to the characteristics inherent of such health plans (refer to EXHIBIT A). This is expected as consumers demand freedom of choice and perceive quality with the degree of freedom provided. However, these conventional plans are costly and, as a result, employers are dropping such options from their benefit programs. In this particular illustration, the University of Pittsburgh has closed their indemnity option this year.

A second super-matrix assesses the shift in employee preferences of health plans in response to this change in options. Without the BC/BS Traditional plan, HealthAmerica is the preferred plan (refer to EXHIBIT B). True value in health care coverage requires a combination of affordable premiums, comprehensive benefits, and low out-of-pocket expenses (among other factors discussed). The rates of increase in the plan premiums tell only part of the story. Benefits offered under the various plans and the level of out-of-pocket costs faced by consumers in each plan not only affect the plan premiums over time, but also largely determine the market shares of the different health plans. Although traditional fee-for-service plans have added benefits over the last five years, HMO plans are still richer in benefits overall. The percentage of employees given the choice of rider benefits such as mental health and substance abuse treatment in traditional plans has increased very slightly, while HMOs continue to offer a comprehensive package of benefits, including both preventive services and rider options. 
Pre-existing condition limitations - clauses that postpone the effective date of coverage for new enroliees' existing health conditions for a period of time after they join the plan - postpone the effective date of coverage under a health plan. This practice discourages employees from switching plans and denies benefits for a specified period of time - still a fairly prevalent practice in traditional and PPO plans. HMOs, on the other hand, do not have such limitations. For instance, approximately $63 \%$ of new traditional fee-for-service enrollees must wait an average of nine months for pre-existing conditions to be covered, and an estimated $72 \%$ of new PPO enrollees must wait an average of ten months for the conditions to be covered ${ }^{6}$.

Because the financial protection of health care coverage depends upon a combination of premiums, benefits, and out-of-pocket expenses, etc., comparisons of the premiums of health plans alone can be misleading. When out-of-pocket expenses such as deductibles and coinsurance are excessively high, even a health plan with an affordable premium may not prove to be adequate financial protection for the typical consumer. HMOs generally provide members comprehensive benefits while requiring only minimal out-of-pocket payments for the use of services. HMO members usually pay a co-payment (between $\$ 2$ and $\$ 10$ for each office visit) and are not generally subject to deductibles or coinsurance. Traditional fee-for-service plans and PPOs, however, often charge deductibles which require consumers to pay the full cost of care until a specified dollar limit is reached. In addition, traditional and PPO plans often charge coinsurance, requiring consumers to pay a percentage of the cost of treatment above the deductible (usually $20 \%$ for par providers and $40 \%$ for non-par providers).

Again, HMOs achieve these cost savings while offering more comprehensive benefits and lower out-of-pocket costs to consumers. For example, more HMOs than fee-for-service plans cover preventive health care services such as adult physicals and well-baby care. In addition, HMOs often require co-payments from enrolled members for each visit, but rarely impose deductibles or coinsurance. Finally, HMOs do not limit their coverage of pre-existing conditions, unlike fee-for-service and PPO plans. Thus, HMO members generally receive more comprehensive benefits at less cost than other plans due to utilization management techniques. With these differences, industry analysts believe that the gap between HMOs and other plans will continue to widen over time until traditional plans are eventually phased out altogether. This will be to the benefit of HMOs, as consumers increasingly accept the attractive elements of these plans and the concept of utilization management. 


\section{WHICH HEALTH PLAN TO CHOOSE? \\ 1992 PARTICIPATION IN HEALTH PLAN OPTIONS \\ UNIVERSITY OF PITTSBURGH PERSONNEL SYSTEM \\ EXHIBIT A}

\begin{tabular}{lc} 
& With BC/BS Traditional Option \\
\cline { 2 - 2 } Traditional & $48.8 \%$ \\
HealthAmerica & $26.8 \%$ \\
Deductible & $13.2 \%$ \\
University Health Network & $7.2 \%$ \\
Keystone West & $4.0 \%$ \\
& EXHIBIT B
\end{tabular}

Super-Matrix Results

$46.4 \%$

$27.1 \%$

$14.5 \%$

$7.5 \%$

$4.5 \%$
Without BC/BS Traditional Option

HealthAmerica

Deductible

University Health Network

Keystone West
$52.5 \%$

$25.7 \%$

$14.0 \%$

$7.8 \%$
Super-Matrix Results

$50.9 \%$

$27.0 \%$

$15.4 \%$

$6.7 \%$

\section{REFERENCES}

${ }^{1}$ S.J. Reiser, "The era of the patient, " JAMA 269 (8): 1012, February 24, 1993.

${ }^{2}$ O.S.J. Mascarenhas, "Marketing health care to employees: The structure of employee health care plan satisfaction," Journal of Health Care Marketing 13 (Fall): 34, 1993.

${ }^{3}$ Trends in Health Insurance, KPMG Peat Marwick, p. 14.

${ }^{4}$ Reiser (cited above at note 1); and T.W. Moloney and B. Paul, "The consumer movement takes hold in medical care," Health Affairs 10 (4):268, Winter 1991.

5J. Gabel, "Employer-Sponsored Health Insurance, 1989" Health Affairs, Fall 1990, p 167.

${ }^{6}$ Trends in Health Insurance, KPMG Peat Marwick, p. 7.

\section{OTHER SOURCES}

The Health Plan Employer Data and Information Set (HEDIS) 2.0 represents a core set of standardized definitions and specific methodologies for deriving and systematizing health plan performance measures, National Committee for Quality Assurance. The Employee Health Care Value Survey by the New England Medical Center and the Group Health Association of America (GHAA) Consumer Satisfaction Survey are also used to assess major concepts in the evaluation of health plans and performance.

Health Care Benefits Survey, A. Foster Higgins, Princeton, NJ, 1992.

Managed Care Survey, Health Insurance Asscociation of America (HIAA), 1991. 